Z Epileptol 2017 · 30:1-2 DOI 10.1007/s10309-016-0101-2

Online publiziert: 2 . Januar 2017

๑) Springer Medizin Verlag Berlin 2016

CrossMark

\author{
F. Bösebeck + A. Strzelczyk $k^{2,3}$ \\ 'Epilepsiezentrum der Neurologischen Klinik, AGAPLESION Diakonieklinikum, Rotenburg, Deutschland \\ ${ }^{2}$ Epilepsiezentrum Frankfurt Rhein-Main Zentrum der Neurologie und Neurochirurgie, Goethe-Universität \\ Frankfurt, Frankfurt am Main, Deutschland \\ ${ }^{3}$ Epilepsiezentrum Hessen, Klinik für Neurologie, Philipps-Universität Marburg, Marburg, Deutschland
}

\title{
The Borderland of Epilepsy
}

Im Jahr 1907 veröffentlichte der englische Nervenarzt Sir William Gowers eine umfangreiche Zusammenstellung der Differentialdiagnosen epileptischer Anfälle unter dem Titel „The Borderland of Epilepsy: Faints, Vagal Attacks, Vertigo, Migraine, Sleep Symptoms and their Treatment", die bereits kurz nach ihrem Erscheinen in London in verschiedene Sprachen (u. a. ins Deutsche) übersetzt wurde $[1,2]$. In dieser historisch wie aktuell häufig zitierten Arbeit wird in einer für die damalige Zeit beeindruckenden Differenziertheit und Präzision über die Pathophysiologie und diagnostische Relevanz von epileptischen und nicht epileptischen Phänomenen berichtet, deren Inhalt in weiten Teilen bis heute noch Gültigkeit besitzt. In dem vorliegenden Themenheft gleichen Namens widmen wir uns einer ausgesuchten Auswahl von Differenzialdiagnosen epileptischer Anfälle aus dem Blickwinkel des gegenwärtigen wissenschaftlichen Kenntnisstandes. Die Inhalte einiger Artikel dieses Themenheftes sind Teil der Vortragsreihe "The Borderland of Epilepsy“, die auf der Jahrestagung der Deutschen Gesellschaft für Epileptologie im März 2016 in Jena präsentiert wurde.

Einleitend würdigen $\mathrm{M}$. Reuber et al. in ihrem Artikel „William Gowers und das,Borderland of Epilepsy" 'zunächst das Leben und Wirken von William Gowers im wissenschaftshistorischen Kontext des ausgehenden 19. Jahrhunderts und beleuchten damit auch die Frühzeit der strukturierten Epilepsiebetreuung in Europa [3].

In seinem Artikel „The Borderland of ictal Phenomenology and Pathophysiolo$g y$ " fokussiert F. Bösebeck zunächst die generelle Problematik der Differenzial- diagnostik episodischer Phänomene in der Neurologie und spannt anschließend einen Bogen von den historischen Konzepten zur Pathophysiologie und zum symptomatischen Phänotypus in der Zeit von Gowers bis zu gegenwärtigen Netzwerktheorien [4].

Der Artikel „Myoklonus - eine Orientierungshilfe"von H.M. Meinckgibt einen allumfassenden Überblick über die Pathophysiologie epileptischer und nicht epileptischer Myoklonien. Darüber hinaus werden klinisch relevante Untersuchungsmethoden und typische Befundkonstellation der verschiedenen myoklonischen Phänomene und Störungen vorgestellt [5].

Eine praktische Hilfe bei der Abgrenzung visueller Auren im Rahmen epileptischer Anfälle von Migräneattacken bietet die Übersicht von A. Strzelczyk und Kollegen. Bei hoher Koinzidenz periiktaler Kopfschmerzen können visuelle Auren epileptischer Genese als Migräneauren fehldiagnostiziert werden, wie ein eindrückliches Fallbeispiel zeigt. Zudem werden mit dem GastautSyndrom und dem PanayiotopoulosSyndrom zwei häufige idiopathische Occipitallappenepilepsien des Kindesalters ausführlich besprochen, die ebenfalls mit visuellen Symptomen und Kopfschmerzen vergesellschaftet sein können [6].

G. Möddel und A. Heidbreder widmen sich nicht epileptischen, motorischen Phänomenen im Schlaf, die eine wichtige Differenzialdiagnose $\mathrm{zu}$ Frontallappenanfällen darstellen. Ihre Übersicht bietet einen Überblick und Hinweise zur Unterscheidung zwischen Non-REM-Parasomnien sowie der REM-Schlaf-Verhaltensstörung. Letztere kann um viele Jahre neurodegenerativen Erkrankungen wie M. Parkinson oder Multisystematrophie vorausgehen. Ein Überblick über periodische Beinbewegungen, Bruxismus, hypnagogen Fußtremor, alternierende Beinmuskelaktivierung im Schlaf sowie Einschlafmyoklonien ergänzt den Beitrag [7].

Der Artikel „Hypothesen zur Genese dissoziativer Anfälle anhand der Anfallsschilderungen" von J. Opp und B. FrankJob fokussiert eine der wohl häufigsten Fehldiagnosen in der Epileptologie und nähert sich der Differenzierung zwischen epileptisch und nicht epileptisch auf sprachanalytischem Weg. Die Autoren stellen dabei linguistische Untersuchungen der Anfallsbeschreibung von Jugendlichen vor, deren Ergebnisse Epileptologen auch im klinischen Alltag eine Erweiterung ihres diagnostischen Armariums bieten [8].

Im klinischen Alltag stellen Synkopen die häufigste und wichtigste Differentialdiagnose zu epileptischen Anfällen dar. In seinem Beitrag zu Synkopen stellt K.M. Klein die verschiedenen Ätiologien von Synkopen vor und beschreibt wichtige Charakteristika, anhand derer die Anamnese in den meisten Fällen bereits eine differenzialdiagnostische Einordnung erlaubt. Auch die verfügbaren diagnostischen Tests, die in unklaren Fällen oder bei kardialen Synkopen erforderlich sind, werden dargestellt. Eine differenzierte Betrachtung der therapeutischen Optionen rundet den Beitrag ab [9].

Die Notwendigkeit einer interdisziplinären Abklärung von Patienten mit transientem Bewusstseinsverlust sowie die Bedeutung des Routine-EKGs verdeutlicht der Beitrag von M.P. Bergmann 
und Kollegen. Die Diagnose einer Torsade-de-pointes-Tachykardie bei LongQT-Syndrom als Ursache von Synkopen ist selten, ein Routine-EKG kann hierfür Hinweise liefern, im vorgestellten Fall konnte diese Diagnose erst durch Aufzeichnung einer repräsentativen Episode im Video-EEG-Monitoring gesichert werden [10].

Im Beitrag von P.S. Zeiner et al. wird an einem Fallbericht die paroxysmale sympathische Hyperaktivität vorgestellt. Infolge einer Schädigung zerebraler autonomer Zentren kommt es zu einer Überaktivität des sympathischen Systems mit paroxysmal auftretenden dystonen Bewegungsmustern, Hypertension, Tachypnoe, Tachykardie, Hyperthermie, Hyperhidrosis. Patienten mit diesem Symptomkomplex stellen auf Intensivstationen sowie in Rehabilitationseinrichtungen differenzialdiagnostische Herausforderungen dar. Hier kann ein EEG in der Abgrenzung zu epileptischen Anfällen hilfreich sein [11].

Wir hoffen, mit der Auswahl der Beiträge dieses Themenheftes ein möglichst breites Interesse am spannenden Thema der epileptologischen Grenzgebiete geweckt zu haben und wünschen Ihnen viel Vergnügen bei der Lektüre.

Frank Bösebeck, Adam Strzelczyk

\section{Korrespondenzadresse}

\section{Dr. med. F. Bösebeck}

Epilepsiezentrum der Neurologischen Klinik, AGAPLESION Diakonieklinikum Eliese-Averdieck-Straß 17, 27356 Rotenburg, Deutschland

Boesebeck@diako-online.de

\section{Prof. Dr. med. A. Strzelczyk, MHBA}

Epilepsiezentrum Frankfurt Rhein-Main

Zentrum der Neurologie und Neurochirurgie, Goethe-Universität Frankfurt

Schleusenweg 2-16, Haus 95, 60528 Frankfurt am Main, Deutschland

strzelczyk@med.uni-frankfurt.de

Interessenkonflikt. F. Bösebeck und A. Strzelczyk geben an, dass kein Interessenkonflikt besteht.

\section{Literatur}

1. Gowers WR (1907) The borderland of epilepsy: faints, vagal attacks, vertigo, migraine, sleep symptoms and their treatment. Churchill, London
2. Gowers WR (1908) Das Grenzgebiet der Epilepsie. Deuticke, Leipzig

3. Reuber M, BösebeckF, OppJ (2017) William Gowers und das „borderland of epilepsy“. Z Epileptol. doi:10.1007/s10309-016-0093-y

4. Bösebeck F (2017) The borderland of ictal phenomenology and pathophysiology. Z Epileptol. doi:10.1007/s10309-016-0100-3

5. Meinck HM (2017) Myoklonus - eine Orientierungshilfe. Z Epileptol. doi:10.1007/s10309-0160087-9

6. Strzelczyk A, Gaul C, Rosenow F, Kurlemann G (2017) Visuelle Auren im Grenzgebiet zwischen Epilepsie und Migräne. Z Epileptol. doi:10.1007/ s10309-016-0098-6

7. Möddel G, Heidbreder A (2017) Nicht-epileptische motorische Phänomene im Schlaf. Z Epileptol. doi:10.1007/s10309-016-0088-8

8. Opp J, Frank-Job B (2017) Hypothesen zur Genese dissoziativer Anfälle anhand der Anfallsschilderungen. Z Epileptol. doi:10.1007/s10309-0160094-x

9. Klein KM (2017) Synkopen. Z Epileptol. doi:10. 1007/s10309-016-0096-8

10. Bergmann MP, Belke M, Lüsebrink U, Knake S, Rosenow F, Strzelczyk A (2017) Differenzialdiagnose paroxysmaler Bewusstseinsstörungen Torsadede-pointes-Tachykardie bei Long-QT-Syndrom. ZEpileptol.doi:10.1007/s10309-016-0089-7

11. Zeiner PS, Meyer-Ohlendorf M, Dvorak F, Jahnke K, Reif PS, Strzelczyk A (2017) Paroxysmale sympathische Hyperaktivität als Syndrom im Grenzgebiet der Epilepsie. Z Epileptol. doi:10. 1007/s10309-016-0083-0

\section{Auch online Zugang zu allen Beiträgen Ihres Individual- abonnements}

Wussten Sie, dass Sie als Abonnent dieser Zeitschrift automatisch online Zugriffsrechte auf das gesamte Beitragsarchiv und die CME-Kurse haben?

Der Zugang zu Ihrer Online-Bibliothek und den CME-Kursen ist im Abonnement Ihrer Zeitschrift inbegriffen. Neben den gedruckten Ausgaben, können Sie Themen einfach am Computer recherchieren. Für den Zugang müssen Sie sich lediglich einmal über www.springermedizin.de/register registrieren.

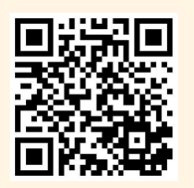

Über diesen QR-Code schnell und einfach registrieren

Für die Registrierung brauchen Sie nur

- die Nummer Ihres Abonnements

(siehe Adressaufkleber auf Ihrem Heft) und

- Ihre Lieferanschrift.

So stellen Sie die Zugehörigkeit zu Ihrer Zeitschrift sicher.

Nach Login erhalten Sie dann sofort die Online-Rechte für den Volltextzugriff und den Zugang zu Ihren CME-Kursen.

Mit Benutzername und Passwort haben Sie außerdem Zugang zu den freien Inhalten auf den Seiten von:

https://www.springermedizin.de/ http://www.aerztezeitung.de/ http://www.heilberufe-online.de/

Sollten Fragen oder Probleme auftauchen, wenden Sie sich einfach an Ihren Kundenservice über:

kundenservice@springermedizin.de 